\title{
Intra-muscular administration of autologous bone marrow mononuclear cells reduces opioid dependency in patients with critical limb threatening ischemia.
}

Justin L. Couetil ${ }^{1}$, Michael P. Murphy ${ }^{2}$

${ }^{1}$ Indiana University School of Medicine; ${ }^{2}$ Indiana University School of Medicine, Department of Vascular Surgery

\section{Background and Hypothesis}

Critical limb-threatening ischemia (CLTI) is a severe limitation in perfusion of the lower extremities. It is the most advanced stage of peripheral arterial disease. Characterized by unremitting rest pain and/or gangrene, the CLTI patient population has an excessively high rate of opioid use and addiction. The MOBILE Trial was a Phase II multi-center, double-blinded placebo controlled trial designed to assess the safety and efficacy of autologous bone marrow cells $(A B M C)$ in treating patients with CLTI. Our central hypothesis is that cell therapy may provide trophic effects in the treated limb that may decrease opioid requirements.

\section{Project Methods}

The primary endpoint of the MOBILE Trial was amputation-free survival at 52 weeks. Secondary endpoints included limb perfusion measures, ambulatory function, quality of life (VascuQol), pain assessment with a visual analog score, and opioid requirements, as tabulated by prescriptions reported to us. A post-hoc statistical analysis using ANOVA was conducted to describe changes in pain and quality of life measures along the study, and Fisher's test to compare the incidence of new opioid prescriptions between treatment groups.

\section{Results}

There were no differences in quality of life and visual analog pain measures between the ABMC and placebo groups $(P=0.42)$. There was however a $36 \%$ decrease in new opioid prescriptions in the ABMC groups as compared to placebo $(P=0.058, H R:-0.44-1.012)$. This reduction did not correlate with changes in limb perfusion measures, gender, age, diagnosis of diabetes, or Rutherford score.

\section{Discussion}

The results of this analysis demonstrate an effect of $A B M C$ in reducing opioid use in a high-risk addiction patient population. These findings are consistent with other studies assessing mesenchymal stem cells in chronic pain syndromes. The effect of ABMC did not correlate with changes in limb perfusion suggesting other beneficial trophic effects of cells in ischemic induced neuropathies. This discovery suggests a potential therapeutic strategy for reducing opioid dependency. 